\title{
The Study of Predictive Value of Uterine Artery Doppler in Incidence of Preeclampsia and Intrauterine Growth Restrictions in Pregnant Women
}

\author{
Sedigheh Borna ${ }^{1}$, Shahla Nasrolahi ${ }^{2}$, Shahedeh Khansari ${ }^{*}$
}

\begin{abstract}
Objectives: Preeclampsia is considered as one of the most important causes of death among pregnant women all around the world. This study aimed to study the predictive value of uterine artery Doppler ultrasonography in the incidence of preeclampsia and the intrauterine growth restriction (IUGR) in pregnant women who attended Fatemieh hospital.

Materials and Methods: This study was performed by the prospective cohort method and the statistical population included 108 pregnant women, in their 18-22 weeks of pregnancy, with a singleton pregnancy who referred to the prenatal Fatemieh Hospital of Hamadan during 2013-2016. The uterine artery Doppler ultrasonography was performed and the incidence of IUGR and the occurrence of preeclampsia in the followed pregnancy was evaluated in these patients. Data were analyzed by SPSS 16.0 using the chi-square and Mann-Whitney U statistical tests.

Results: The mean difference of pulsatility index (PI) in patients with preeclampsia was statistically significant compared to healthy individuals $(P=0.001)$. In addition, the mean difference of PI in individuals with infants suffering from IUGR was statistically significant compared with the infants not suffering from the IUGR $(P=0.001)$. Further, the incidence of preeclampsia and IUGR in women with a bilateral notch in ultrasonography was significantly greater than those of the women with no report of a bilateral notch in ultrasonography $(P=0.001)$.

Conclusions: Therefore, Doppler ultrasonography of the uterine artery has a predictive value in preeclampsia among pregnant women.

Keywords: Doppler ultrasonography, Uterine artery, Preeclampsia, Intrauterine growth restriction
\end{abstract}

\section{Introduction}

Hypertensive disorders commonly occur during pregnancy and are considered as three fatal factors when combined with bleeding and infection. These factors are responsible for the major part of mortalities and maternal complications including preeclampsia (1-3). According to the estimation of the World Health Organization, more than one million women around the world annually die due to preeclampsia and its complication which lead to maternal deaths in developing countries. This rate is 5-10 and more than $18 \%$ in developing countries and the developed countries, respectively $(4,5)$.

In addition, preeclampsia is one of the pregnancyspecific disorders in pregnancy, which is characterized by hypertension and proteinuria after 20 weeks of pregnancy. Proteinuria is an important sign of preeclampsia and contains the protein excretion of $300 \mathrm{mg}$ or more within 24 hours or persistent proteinuria of $30 \mathrm{mg}$ per deciliter in random urine samples $(2,6,7)$. Further, preeclampsia often occurs in young and nulliparous women and its incidence is greatly influenced by race, ethnicity, and genetic predisposition.

Other factors include the environmental, social, economic, and even seasonal effects, as well as maternal obesity, multiple pregnancy, older age, and the history of hypertension in the previous pregnancy (8-10).

The exact pathophysiology of this disease is not clear, but it is shown that preeclampsia is associated with an imbalance between the production of prostacyclin, thromboxane A2, and vascular spasm (11). The end organ involvement in the form of heart failure, pulmonary edema, liver involvement, and coagulation system, and finally, the hemolysis, elevated liver enzymes, and low platelet count (HELLP) syndrome are considered as its serious complications. Furthermore, preeclampsia is accompanied by a decline in renal function, cerebral involvement, convulsions, and even maternal deaths. On the other hand, the incidence of intrauterine growth restriction (IUGR) and intrauterine fetal death is also possible due to a problem in placental perfusion $(12,13)$.

The Doppler ultrasonography, as a non-invasive technique, can be used to investigate placental blood 
flow. The impedance of uterine artery flow progressively reduces during the first and second trimester of normal pregnancy due to the trophoblastic invasion of the musculo-elastic membrane of uterine spiral arteries (14). Moreover, the pathology of the placenta in pregnancies with preeclampsia and IUGR indicates the failure in the normal transmission of maternal placental arteries to the vessels with low resistance $(15,16)$.

Some studies expressed that an abnormal Doppler at 11-14 weeks of pregnancy can be effective in identifying the high-risk patients in preeclampsia $(17,18)$. Abnormal findings in Doppler ultrasonography include a unilateral or bilateral notch in the diastolic notch or vascular resistance index (RI) or high pulsatility index (PI) is high $(19,20)$. Espinoza et al conducted a study to identify patients at the risk of severe or mild preeclampsia using uterine arteries Doppler. They found that abnormal uterine artery Doppler and abnormal placental growth factor (PGF) are 2 independent risk factors for the occurrence of severe and mild pre-eclampsia, as well as IUGR (21). Similarly, the results of the study by Myatt et al showed that the incidence of preeclampsia was significantly correlated with higher RI and the PI was higher than 0.75 in patients with preeclampsia (22). Dehghani-Firouzabadi et al indicated that the average ratio of RI in women with infants having IUGR was significantly higher than that of the other groups (23). The present study sought to investigate the predictive value of uterine artery Doppler ultrasonography in the incidence of preeclampsia and IUGR in those pregnant women referring to prenatal Fatemieh hospital of Hamedan during 2013-2016.

\section{Materials and Methods}

This study was performed using a prospective cohort method in order to evaluate the predictive value of uterine artery Doppler ultrasonography in the incidence of preeclampsia and IUGR in pregnant women.

The population of the study included all pregnant women, in 18 to 22 weeks of pregnancy, with singleton pregnancies who referred to the prenatal clinic of Fatemieh hospital during 2013-2016. The exclusion criteria included patients with advanced underlying disease, diabetes, and advanced liver and kidney diseases.

The sample selection was conducted by census method and among the eligible patients, those who had the exclusion criteria underwent a survey. Additionally, after being justified to the study and its objectives, 108 patients obtained the participation consent for the study. Then, all patients underwent uterine artery Doppler ultrasonography. Uterine artery Doppler was conducted in the semi-upright position with a probe, as an operator for all patients.

Ultrasonography was performed by probe Convex 3.5 of Emedicine $\mathrm{x} 10$ device in the junction between uterine arteries and external iliac. In all the patients, the PI of every uterine artery was calculated by averaging 3 consecutive waves using the formula of the maximum systolic volume difference of end-diastolic volume divided by the average volume. In addition, a unilateral or bilateral notch was recorded in a diastolic wave, if present.

Then, all patients were followed and the occurrence or non-occurrence of preeclampsia in their pregnancy was evaluated in these patients. Further, the incidence or non-incidence of intrauterine growth was investigated as a weight less or more than 10 . Then, all the information related to patients was included in the checklist specific to each patient, such as demographic data (i.e., age and sex), persistence index, the unilateral or bilateral notch in the diastolic notch, and the presence or absence of preeclampsia or IUGR. Finally, all data entered into SPSS software, version 16 and data were described using descriptive and inferential statistics indices, and finally, statistically analyzed using the chi-square and MannWhitney statistical tests. The significance level was considered below 0.05 .

Limitations of the study included the lack of follow-up visits of the patients, therefore, their telephone numbers were taken and followed for solving this issue.

\section{Results}

The average age of pregnant mothers was 28.00 with an age range of 17 to 43 years. Furthermore, the mean number of pregnancies in the pregnant women was 1.78 with a range of 1 to 5 and the average weight of the newborns at the time of birth was $3069.88 \mathrm{~g}$ with a range of 1200 to $4100 \mathrm{~g}$. Moreover, the mean time of ultrasonography in pregnant women with 19.45 weeks of pregnancies ranged from 18 to 22 weeks.

The 2-week delay at the time of ultrasonography relative to specified time was due to the time of patients' admission and restrictions in the number of studied patients. The average left plasticity index in pregnant women was 1.17 of the wavelength with a range of 0.35 to 2.88 and the average right PI was 1.19 of the wavelength ranging from 0.35 to 2.91 . In total, the mean right and left plasticity index in the participants was 1.18 with a range of 0.45 to 2.77 of a wavelength.

In the studies of intrauterine artery Doppler ultrasonography, 8 patients $(7.4 \%)$ out of 108 pregnant women participated in the study had a right-hand notch whereas 9 patients $(8.3 \%)$ had left notch and 10 patients had both left and right notches. However, no notch was observed in the ultrasonography of the other 81 patients (75\%). The mean of both left and right PI was $\geq 1.5$ in 28 patients while 80 patients $(74.1 \%)$ had the mean PI $<1.5$ of a wavelength, in which the incidence of preeclampsia was less. In the follow-up studies of the participated patients, $64(59.3 \%)$ and $44(40.7 \%)$ out of 108 pregnant women had normal and caesarean section deliveries, respectively. In addition, $11(10.2 \%)$ and $16(14.8 \%)$ patients had severe hypertension and non-severe hypertension, respectively. However, no sign of gestational hypertension was observed 
Table 1. Comparison of Notch in the Sonography of Patients With and Without Preeclampsia and Neonates With and Without IUGR

\begin{tabular}{lcccc}
\hline Notch & $\begin{array}{c}\text { Not With } \\
\text { IUGR }\end{array}$ & IUGR & $\begin{array}{c}\text { Not With } \\
\text { Preeclampsia }\end{array}$ & Preeclampsia \\
\hline No & $76(78.3)$ & $5(45.4)$ & $66(82.5)$ & $15(53.6)$ \\
Right & $7(7.2)$ & $1(9.1)$ & $6(7.5)$ & $2(7.1)$ \\
Left & $9(9.3)$ & $0(0.00)$ & $6(7.5)$ & $3(10.7)$ \\
Both sides & $5(5.6)$ & $5(45.6)$ & $2(2.5)$ & $8(28.6)$ \\
Significance & \multicolumn{2}{c}{0.001} & \multicolumn{3}{c}{0.001} \\
\hline
\end{tabular}

IUGR, Intrauterine growth restriction.

in the remaining 81 patients. Likewise, in terms of having proteinuria, $6,7,3$, and 2 patients suffered from $1+, 2+$, $3+$, and $4+$ proteinuria, respectively. Additionally, four of these patients were hospitalized for further diagnosis and treatment, from whom 24-hour urine samples were taken. However, no sign of proteinuria was detected in the other 90 patients $(83.3 \%)$ during pregnancy. Of the total number of newborns of the study, 11 patients (10.2\%) had IUGR, that is, their weights were less or more than 10 .

The mean PI in the patients with preeclampsia was 1.83 while it had a value of 0.95 of wavelength in nonpreeclamptic patients, which indicates a meaningful difference between the patients in this regard $(P=0.001)$.

In addition, the mean PI in women having infants with and without IUGR was 1.74 and 1.11 of wavelength, respectively, representing a statistically meaningful difference among the women $(P=0.001)$ (Figure 1$)$.

According to the findings of ultrasonography, the incidence of preeclampsia in women, with reported bilateral notch, was meaningfully more than its incidence in women with no report of a notch $(P=0.001)$. Further, the incidence of IUGR in the infants of women with bilateral notches was significantly higher than that of the infants of women with no notch in ultrasonography $(P=0.001)$ (Table 1 and Figure 2).

It is noteworthy that the pregnancy prematurely terminated in 15 patients of the study for various reasons. Furthermore, 2 preterm deliveries were observed, in one of which the fetus was delivered by cesarean section due to being breech (Table 2).

The test results of multivariable logistic regression analysis showed that the chance of preeclampsia was 6.08 times higher in patients with a mean PI of more than 1.5 than those with the mean PI less than 1.5 in the identical condition in terms of having a notch, demonstrating that the difference between these two groups was statistically significant. However, in patients with a bilateral notch in ultrasonography, the chance of preeclampsia was 4.22 times higher than those without a bilateral notch in terms of PI in identical conditions. Thus, the high PI in the Doppler ultrasonography of the uterine artery is a significant predictive factor for preeclampsia (Table 3 and Figure 3).

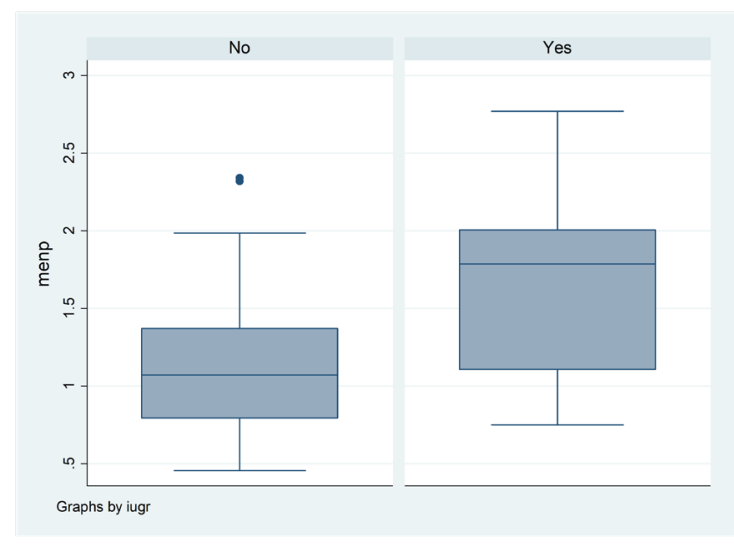

Figure 1. Box Plot of Pulsatility Index in Women Having Children With IUGR or Healthy Babies.

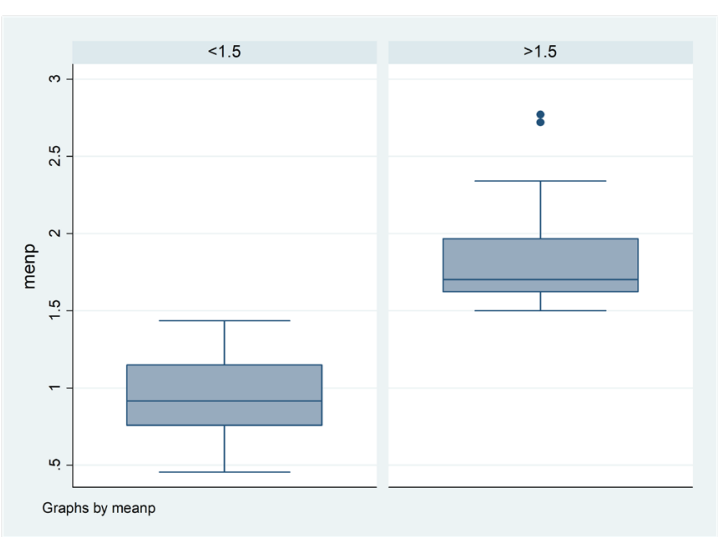

Figure 2. Box Plot of Pulsatility Index in Patients With Preeclampsia $(<1.5)$ and Healthy People.

Table 2. Distribution of Causes of Premature Termination of Pregnancy in Seventeen Cases of the Studied Subjects

\begin{tabular}{lcc}
\hline Causes of Pregnancy Termination & No. & $\%$ \\
\hline Preeclampsia & 6 & $35 / 29$ \\
Preeclampsia and thrombocytopenia & 2 & 11.76 \\
High blood pressure, chronic hypertension, and & 2 & 11.76 \\
consumption of methyldopa & & \\
Preeclampsia and high liver function test & 1 & 5.88 \\
Preeclampsia, chronic hypertension, and & 1 & 5.88 \\
consumption of methyldopa & & \\
High blood pressure & 1 & 5.88 \\
Cholestasis and increased aspartate & 1 & 5.88 \\
aminotransferase and alanine aminotransferase & & \\
Rheum & 1 & 5.88 \\
Preterm delivery & 2 & 11.76 \\
Total & 17 & 100 \\
\hline
\end{tabular}

\section{Discussion and Conclusions}

Preeclampsia is an abnormality in blood pressure that occurs in the course of pregnancy and is characterized by reduced organ perfusions following severe vascular vasospasm (1). The World Health Organization estimated that over one million women in the world die due to 
Table 3. Multivariate Regression Analysis of Predictive Factors for Preeclampsia

\begin{tabular}{llccc}
\hline \multirow{2}{*}{ Variable } & \multirow{2}{*}{ OR } & \multicolumn{2}{c}{$95 \% \mathrm{Cl}$} & \multirow{2}{*}{$\boldsymbol{P}$ Value } \\
\cline { 3 - 4 } & & Low Level & High Level & \\
\hline $\mathrm{PI}$ & 6.08 & 17.27 & 2.13 & 0.001 \\
Without notch & Reference & - & - & - \\
Unilateral notch & 1.55 & 0.43 & 5.6 & 0.497 \\
Bilateral notch & 4.22 & 0.83 & 21.36 & 0.082 \\
\hline
\end{tabular}

OR: odds ratio; $\mathrm{PI}$ : pulsatility index.

preeclampsia and its complications each year and maternal deaths in developing countries is due to this situation $(4,5)$. Moreover, preeclampsia usually occurs in young and nulliparous women and its incidence is greatly influenced by race, ethnicity, and genetic predisposition $(8,9)$. Doppler ultrasonography is a non-invasive technique which can be utilized for assessing uterus-placental blood flow (14). Some studies suggested that an abnormal Doppler in the first trimester of pregnancy can be effective in identifying those at the high risk of having preeclampsia $(17,18)$.

The present study attempted to assess the predictive value of uterine artery Doppler ultrasonography in the incidence of preeclampsia and the IUGR in pregnant women referring to the prenatal Fatemieh clinic of Hamedan during 2013-2016.

In the prospective cohort study, 108 pregnant women with a mean age of 28 years underwent uterine artery Doppler ultrasonography at 18 to 22 weeks of pregnancy. In the study by Barzin et al (24), the transabdominal uterine artery Doppler ultrasonography was conducted on 100 pregnant women with a mean age of 23.2 from 11 to 14 and 21 to 24 of gestational weeks. In another study by Dehghani-Firouzabadi (23), uterine artery Doppler ultrasonography was performed at 14 to 16 weeks of pregnancy. Similarly, Papageorghiou et al (25) used uterine artery Doppler ultrasonography for the patients through a transvaginal method at 23 gestational weeks for predicting the incidence of preeclampsia.

Based on the follow-up investigation of 108 pregnant women of the current study, $11(10.2 \%)$ and $16(14.8 \%)$ patients were diagnosed with severe and non-severe hypertension, but no sign of gestational hypertension was observed in the other 81 patients.

In terms of having proteinuria, $6,7,3$, and 2 patients suffered from $1+, 2+, 3+$, and $4+$ proteinuria, respectively. In the other 90 patients ( $83.3 \%$ ), no sign of proteinuria was observed during pregnancy. Barzin et al (24) found that 10 patients from the studied population had preeclampsia, out of whom 2 and 8 patients suffered from severe and mild preeclampsia, respectively.

Likewise, Papageorghiou et al (25) studied a total of 8335 pregnant women and observed preeclampsia in 71 of these patients. In addition, the IUGR was detected in 698 patients $(9.8 \%)$ in their study. In our study, IUGR was finally observed in 11 patients of the total number of newborns (10.2\%).

In the presentstudy, the mean left and right PIs in pregnant women were 1.17 and 1.19 times of the wavelength with a range of 0.35-2.88 and 0.35-2.91, respectively. Overall, the mean right and left PI in the participants was 1.18 with a range of $0.45-2.77$ of the wavelength and among 108 studied subjects, the mean PI (left and right) of 28 patients was $\geq 1.5$ while that of 80 patients $(74.1 \%)$ was less than 1.5 of a wavelength and thus the incidence of preeclampsia was less probable. In the study by Papageorghiou et al (25), of 8335 pregnant women, 95\% had a high PI. Further, Barzin et al (24) reported the mean PI of 1.84 and 0.82 in the weeks of 11-14 and 21-24, respectively. Finally, Barzin et al found that the sensitivity and specificity of PI for the diagnosis of preeclampsia were greater in the second trimester compared to the first trimester. In our study, the mean PI in patients with preeclampsia was 1.83 while it was equal to 0.95 of a wavelength in terms of having preeclampsia in healthy subjects. Thus, the mean PI difference between patients with preeclampsia and healthy individuals was statistically significant regarding pre-eclampsia. Furthermore, the mean PI in patients with infants with IUGR was equal to 1.74 while that of those with infants without IUGR was equal to 1.11 of a wavelength, indicating that the difference between them was significant in those with restriction. Therefore, the mean PI difference between the patients having infants with or without IUGR was statistically significant. In the study by Myatt et al, the pulsatile index was above 0.75 in patients with preeclampsia (22). Moreover, Papageorghiou et al (25) reported that of all women whose infants had IUGR (9.8\%), 69\% had a high PI. In addition, the results of the study by Espinoza et al (21) showed that abnormal uterine artery Doppler, along with an abnormal PI are two independent risk factors for severe and mild preeclampsia and IUGR.

In the present study, the uterine artery Doppler ultrasonography of women indicated that of 108 pregnant women participating in the study, 8 (7.4\%), 9 (8.3\%), and 10 patients had right, left, and simultaneous left and right notches, respectively. Barzin et al (24) concluded that the pre-diastolic notch values in patients with preeclampsia and women with normal pregnancy were $80 \%$ and $78 \%$ in the first trimester, respectively. However, in the assessment of second trimester (weeks 21-24), the notch was observed in $17 \%$ of the women with normal pregnancies while notches were found in $60 \%$ of patients with preeclampsia. Examining the incidence of preeclampsia according to the results of ultrasonography, it was revealed that the incidence of preeclampsia in women with the detected bilateral notch was significantly higher compared to women without a notch in the current study.

Similarly, investigating the incidence of IUGR according to the results of the ultrasonography, the incidence of IUGR was significantly higher in the infants of women with a bilateral notch as compared to the infants of women 
with no report of a notch in their ultrasonography. Finally, Barzin et al (24) also considered bilateral notch and the high mean PI in Doppler ultrasonography as a useful standard method in the diagnosis of preeclampsia in high-risk pregnancies. In addition, based on the result of the present study, the chance of having preeclampsia was 6.08 times more in patients with a mean PI of higher than 1.5 compared to patients with a mean PI of less than 1.5 in the identical condition. Additionally, in patients with a bilateral notch in the ultrasonography, the chance of preeclampsia incidence was 4.22 times higher than those without a bilateral notch in the identical conditions in terms of PI. According to the results of another study (26), the combination of uterine artery PI and serum HRG level at 11-13+6 weeks of gestation was not an effective method for predicting preeclampsia at the time of firsttrimester screening. However, considering the findings of the above-mentioned study, it can be concluded that there is a significant relationship between the findings of uterine artery Doppler ultrasonography and the incidence of preeclampsia and IUGR in pregnant women. In other words, high PI in the uterine artery Doppler ultrasonography is a meaningful predictive factor for preeclampsia incidence. Thus, the uterine artery Doppler ultrasonography at 18 and 22 weeks of pregnancy can be used as an appropriate method for screening the highrisk pregnant patients and prevent irreversible pregnancy complications such as IUGR and intrauterine fetal deaths.

\section{Conflict of Interests}

Authors declare that they have no conflict of interests.

\section{Ethical Issues}

This study was approved by the Ethical Committee of Hamadan University of Medical Sciences in this study (IR. UMSHA.REC.1391.12).

\section{Financial Support}

This study was supported by the vice chancellator for research and technology of Hamadan University of Medical Sciences.

\section{References}

1. Cunningham F, Leveno K, Bloom S, Spong CY, Dashe J. Williams Obstetrics. 24th ed. McGraw-Hill; 2014.

2. Duley L. Pre-eclampsia, eclampsia, and hypertension. BMJ Clin Evid. 2100;2100:1402.

3. Paridaens H, Gruson D. Pre-eclampsia: overview on the role of biomarkers in 2016. Ann Biol Clin (Paris) 2017;75:245-58. doi: 10.1684/abc.2017.1240

4. Bujold E, Morency AM, Roberge S, Lacasse Y, Forest JC, Giguere Y. Acetylsalicylic acid for the prevention of preeclampsia and intra-uterine growth restriction in women with abnormal uterine artery Doppler: a systematic review and meta-analysis. J Obstet Gynaecol Can. 2009;31:818-26.
5. Komacki J, Skrzypczak J. The use of Doppler in the second half of pregnancy. Ginekologia Polska. 2015; 86:626-30.

6. Anderson UD, Gram M, Akerstrom B, Hansson SR. First trimester prediction of preeclampsia. Curr Hypertens Rep. 2015;17:584. doi: 10.1007/s11906015-0584-7

7. Brodowski L, Burlakov J, Hass S, von Kaisenberg C, von Versen-Hoynck F. Impaired functional capacity of fetalendothelial cells in preeclampsia. PloS One. 2017;12:e0178340. doi: 10.1371/journal. pone. 0178340 .

8. Jadli A, Sharma N, Damania K, et al. Promising prognostic markers of preeclampsia: new avenues in waiting. Thromb Res. 2015;136(2):189-95. doi: 10.1016/j.thromres.2015.05.011.

9. Chanthasenanont A, Somprasit C, Pongrojpaw D. Prediction of perinatal outcomes in patient with preeclampsia: maternal hyperoxygenation test on fetal Doppler flow. J Med Assoc Thai 2009;92:161-6.

10. Aghasadeghi F, Saadat M. Association between ABO and $\mathrm{Rh}$ blood groups and risk of preeclampsia: a casecontrol study from Iran. Open Access Maced J Med Sci 2017;5:173-6. doi: 10.3889/oamjms.2017.002

11. Prefumo F, Fratelli N, Ganapathy R, Bhide A, Frusca T, Thilaganathan B. First trimester uterine artery Doppler in women with previous pre-eclampsia. Acta Obstet Gynecol Scand. 2008;87:1271-5. doi: 10.1080/00016340802460347

12. Brunelli VB, Prefumo F. Quality of first trimester risk prediction models for pre-eclampsia: a systematic review. BJOG. 2015;122:904-14. doi: 10.1111/14710528.13334

13. Roberts JM, Escudero C. The placenta in preeclampsia. Pregnancy Hypertens 2012;2:72-83. doi: 10.1016/j. preghy.2012.01.001

14. Prefumo F, Sebire NJ, Thilaganathan B. Decreased endovascular trophoblast invasion in first trimester pregnancies with high-resistance uterine artery Doppler indices. Human Reprod. 2004;19:206-9.

15. Pijnenborg R, Anthony J, Davey DA, et al. Placental bed spiral arteries in the hypertensive disorders of pregnancy. Br J Obstet Gynaecol. 1991;98:648-55.

16. Aardema MW, Oosterhof H, Timmer A, van Rooy I, Aarnoudse JG. Uterine artery Doppler flow and uteroplacental vascular pathology in normal pregnancies and pregnancies complicated by preeclampsia and small for gestational age fetuses. Placenta. 2001;22:405-11. doi: 10.1053/plac.2001.0676

17. Pilalis A, Souka AP, Antsaklis P, Basayiannis K, Benardis P, Haidopoulos D, et al. Screening forpreeclampsia and small for gestational age fetuses at the 11-14 weeks scan by uterine artery Dopplers. Acta Obstet Gynecol Scand. 2007;86(5):530-4.

18. Moon M, Odibo A. First-trimester screening for preeclampsia: impact of maternal parity on 
modeling and screening effectiveness. J Matern Fetal Neonatal Med 2015;28:2028-33. doi: $10.3109 / 14767058.2014 .978758$

19. Bower S, Bewley S, Campbell S. Improved prediction of preeclampsia by two-stage screening of uterine arteries using the early diastolic notch and color Doppler imaging. Obstet Gynecol. 1993;82:78-83.

20. Tayyar A, Guerra L, Wright A, Wright D, Nicolaides $\mathrm{KH}$. Uterine artery pulsatility index in the three trimesters of pregnancy: effects of maternal characteristics and medical history. Ultrasound Obstet Gynecol. 2015;45:689-97. doi: 10.1002/ uog. 14789

21. Espinoza J, Romero R, Nien JK, et al. Identification of patients at risk for early onset and/or severe preeclampsia with the use of uterine artery Doppler velocimetry and placental growth factor. Am J Obstet Gynecol. 2007;196:326.e1-e13.

22. Myatt L, Clifton RG, Roberts JM, et al. The utility of uterine artery Dopplervelocimetry in prediction of preeclampsia in a low-risk population.
Obstet Gynecol. 2012;120:815-22. doi: 10.1097/ AOG.0b013e31826af7fb

23. Dehghani-firouzabadiRazieh M, Saedeh A, Reza N-M. Uterine Artery Doppler Sonography in Predicting Preeclampsia andIUGR at 14-16 Week Gestation. World Applied Sciences Journal 2013;22:197-201.

24. Barzin M, Gholami Z, Erfani A, Bahari M, Hashemi SH. Determination of Prognostic Value of Ureteral Artery Sonography for Pre-eclampsia in Pregnant Women. Journal of Mazandaran University of Medical Sciences. 2015;25:10-8. [Persian].

25. Papageorghiou A, Yu C, Bindra R, Pandis G, Nicolaides K. Multicenter screening for pre-eclampsia and fetal growth restriction by transvaginal uterine artery Doppler at 23 weeks of gestation. Ultrasound Obstet Gynecol. 2001;18(5):441-9.

26. Ramos-Orosco EJ1 et al. Comment on first trimester maternal serum analytes and second trimester uterine artery doppler in the prediction of preeclampsia and fetal growth restriction. Taiwan J Obstet Gynecol. 2018;57(1):175-176. doi: 10.1016/j.tjog.2017.12.033.

(c) 2019 The Author (s); This is an open-access article distributed under the terms of the Creative Commons Attribution License (http://creativecommons.org/licenses/by/4.0), which permits unrestricted use, distribution, and reproduction in any medium, provided the original work is properly cited. 